\title{
Radiation Characteristics of Cavity Backed Aperture Antennas in Finite Ground Plane Using the Hybrid FEM/ MoM Technique and Geometrical Theory of Diffraction
}

\author{
C. J. Reddy ${ }^{1}$, M.D. Deshpande ${ }^{2}$, C.R. Cockrell ${ }^{3}$ and F.B. Beck ${ }^{3}$
}

\begin{abstract}
:
A technique using hybrid Finite Element Method (FEM)/Method of Moments (MoM), and Geometrical Theory of Diffraction (GTD) is presented to analyze the radiation characteristics of cavity fed aperture antennas in a finite ground plane. The cavity which excites the aperture is assumed to be fed by a cylindrical transmission line. The electromagnetic (EM) fields inside the cavity are obtained using FEM. The EM fields and their normal derivatives required for FEM solution are obtained using (1) the modal expansion in the feed region and (2) the MoM for the radiating aperture region(assuming an infinite ground plane). The finiteness of the ground plane is taken into account using GTD. The input admittance of open ended circular, rectangular, and coaxial line radiating into free space through an infinite ground plane are computed and compared with earlier published results. Radiation characteristics of a coaxial cavity fed circular aperture in a finite rectangular ground plane are verified with experimental results.
\end{abstract}

1. C.J.Reddy was previously a National Research Council Research Associate assigned to NASA-Langley Research Center, Mail Stop 490, NASA-Langley Research Center, Hampton VA 23681, USA. Currently he is with Hampton University, Hampton VA 23668, USA.

2. M.D.Deshpande is with ViGYAN Inc., Research Drive, Hampton VA 23681, USA.

3. C.R.Cockrell and F.B.Beck are with NASA-Langley Research Center, Mail Stop 490, Hampton VA 23681, USA. 


\section{INTRODUCTION:}

Cavity backed aperture antennas are useful in aerospace applications due to their nature to be conformal to the surface of the host object[1]. To predict the input impedance and radiation pattern of these types of antennas, one may use the Method of Moments. However, application of MoM to these problems result in full dense matrix and hence for complex cavity structure MoM will require prohibitively large computer storage and CPU time. Alternatively, Finite Element Method (FEM) has been more attractive for the analysis of these problems because FEM has a relatively simple formulation for complex penetrable structures. Also, use of FEM results in sparse, banded matrices, which can be effectively stored and solved. However, FEM for unbounded problems when used alone does not incorporate the Sommerfield radiation condition. The radiation conditions at the boundaries of discretized region must be imposed by using appropriate absorbing boundary conditions. Recent efforts have concentrated on the use of absorbing boundary conditions close to the radiating antenna to reduce the discretization region[2]. Unfortunately, the accuracy of these approximate boundary conditions depends on specific problems, leading to results of unpredictable accuracy. To eliminate the disadvantages of MoM and FEM methods, a combined approach using FEM method inside the cavity and MoM outside the cavity has been proposed $[3,4,5]$ by several authors.

Using the combined FEM/MoM techniques, the input impedances of cavity backed microstrip antennas have been calculated in [1]. The modelling of coaxial feed in [1] is done by replacing the feed probe by a constant filament of current with a delta gap generator. The feed modelling in [1] is expected to give good results for shallow 
cavities. However, for deep cavities rigorous feed modelling is required. In this paper a combined Finite Element Method(FEM) and Method of Moments(MoM) approach with detail feed modelling is used to predict characteristics of cavity backed antennas in infinite ground plane. Various feed lines like rectangular, circular and coaxial waveguides exciting aperture through a cavity are considered. The electromagnetic fields inside the cavity are calculated using the FEM formulation. The boundary values of the field and their normal derivatives required in the FEM solution are obtained by using the waveguide modal expansion function in the feed waveguide region and in the aperture region by using a global method such as the method of moments. Using the present method input admittance of rectangular, circular, and coaxial apertures in an infinite ground plane and excited, respectively, by rectangular, circular, and coaxial transmission lines are calculated and compared with earlier results.

In most antenna measurements, an infinite ground plane is simulated by a relatively large finite ground plane. The change in the radiation pattern of an aperture antenna due to a finite ground plane can be determined using the Geometrical Theory of Diffraction(GTD) [6]. In the implementation of GTD, the radiated fields from the aperture antenna are first determined assuming an infinite ground plane. These fields are used to calculate the incident fields on the edges of finite ground plane. Employing the compact edge diffraction coefficients given in [7] and the incident field on the edges, the diffracted field in the forward as well as backward directions is calculated using the procedure given in [6]. The total radiation field is then obtained by superposition of far field with infinite ground plane assumption and the diffracted field. To verify the validity of the GTD technique when used in combination with FEM/MoM technique, the radiation 
patterns of a coaxial cavity with finite ground plane are calculated and compared with experimental results. Numerical data for input return loss of such a coaxial cavity is also computed and compared with experimental data.

\section{THEORY}

The geometry of the problem to be analyzed is shown in figure 1 . The electric field inside the cavity satisfies the vector wave equation

$$
\nabla \times\left(\frac{1}{\mu_{r}} \nabla \times \vec{E}\right)-k_{o}^{2} \varepsilon_{r} \vec{E}=0
$$

where $\mu_{r}, \varepsilon_{r}$ are the relative permeability and relative permittivity of the medium in the cavity. The electric field $\vec{E}$ is solved via hybrid FEM/MoM technique assuming an infinite ground plane. Following the procedure described in [1,3], equation (1) can be written as

$$
\begin{aligned}
\iiint_{V} \frac{1}{\mu_{r}}(\nabla \times \vec{T}) \cdot(\nabla \times \vec{E}) d v-k_{o}^{2} \varepsilon_{r} \iiint_{V} \vec{T} \bullet \vec{E} d v= & j \omega \mu_{o} \iint_{S_{a}}(\vec{T} \times \hat{n}) \bullet \vec{H}_{a p} d s \\
& +\quad j \omega \mu_{o} \iint_{S_{i n p}}(\vec{T} \times \hat{n}) \bullet \vec{H}_{i n p} d s
\end{aligned}
$$

where $S_{a}$ is the aperture surface in the infinite ground plane and $S_{i n p}$ is the common surface between input feed line and the cavity as shown in figure $1 . \vec{H}_{a p}$ is the magnetic field over the aperture $S_{a}$ and $\vec{H}_{i n p}$ is the magnetic field over the surface $S_{i n p} . \vec{T}$ is the vector testing function. 
In order to solve equation (2) for $\vec{E}$, the volume of the cavity is subdivided into small volumetric tetrahedral elements. Inside a tetrahedral element the electric field may be expressed in terms of the vector edge basis functions[5].

Selecting the testing function $\vec{T}$ to be identical to the basis functions, the left hand side of the equation (2) may be written in a matrix form as

$$
[Z] \cdot[e]
$$

where the matrix $[Z]$ is a symmetrical sparse matrix, and $[e]$ is the column matrix with unknown coefficients for vector basis functions as its elements.

The integeral over the radiating aperture $S_{a}$ is evaluated by finding the electromagnetic field outside the aperture by replacing the aperture with magnetic current $\vec{M}$, backed by an infinite ground plane[1]. The discretization of cavity volume into tetrahedral elements automatically results in discretization of the aperture into triangular elements at $\mathrm{z}=0$ plane. The integral is then evaluated over all the triangles on the aperture surface using the procedure outlined by Rao et al[8]. It can be written in a matrix form as

$$
\left[Z_{a}\right] \cdot\left[e_{a}\right]
$$

where $\left[Z_{a}\right]$ is full dense matrix and $\left[e_{a}\right]$ is a column matrix whose elements are the unknowns amplitudes of electric field over the aperture.

$\vec{H}_{\text {inp }}$ required in the evaluation of the second term on the right hand side of equation (2) can be determined using waveguide vector modal functions. For a waveguide feed, the transverse magnetic field in the feed line can be formulated as the 
incident field and reflected fields as

$$
\vec{H}_{i n p}=\vec{H}_{i n}-\sum_{m} a_{m} \vec{h}_{m}(x, y) Y_{m} e^{\gamma_{m} z}
$$

where $\vec{h}_{m}(x, y)$ is the waveguide vector modal function as defined in [9], $a_{m}$ is the reflection coefficient at the feed line and cavity interface, $\gamma_{m}$ is the propagation constant, $Y_{m}$ is the modal admittance, and $\vec{H}_{\text {in }}$ is the incident field. With the substitution of equation (5), the second term on the right hand side of equation (2) can be written in matrix form as

$$
\left[b_{i n p}\right]-\left[Z_{i n p}\right] \cdot\left[e_{i n p}\right]
$$

For a detailed derivation of equation (6), the reader is referred to [10]. Using equations (3), (4), and (6), equation (2) may be written as

$$
[Z] \cdot[e]-\left[Z_{a}\right] \cdot\left[e_{a}\right]+\left[Z_{i n p}\right] \cdot\left[e_{i n p}\right]=\left[b_{i n p}\right]
$$

Since the column matrices $\left[e_{a}\right]$ and $\left[e_{i n p}\right]$ are the part of $[e]$ all $\mathrm{Z}$ matrices can be combined. The equation (7) then can be written as

$$
[A][e]=\left[b_{i n p}\right]
$$

where $[A]$ is a partly sparse (due to FEM) and partly dense (due to MoM and mode matching). $\left[b_{i n p}\right]$ is the excitation vector and $[e]$ is the unknown vector to be solved. The biconjugate gradient algorithm is used with diagonal preconditioning [11] to efficiently solve equation (8). This algorithm also makes use of the symmetry of the matrix to effectively store the matrix. 
Using the equation (5) the reflection coefficients of any waveguide mode can be obtained.

$$
\Gamma=a_{m}
$$

The input admittance is then calculated as

$$
Y_{i n}=\frac{1+\Gamma}{1-\Gamma} Y_{o}
$$

where $Y_{o}$ is the characteristic admittance of the feed transmission line.

Once the electric field in the aperture is known, the magnetic field at the far field can be evaluated directly from the equation:

$$
\vec{H}_{f f}(r, \theta, \phi)=-\frac{j k_{o}}{\eta_{o}} \frac{e^{-j k_{o} r}}{2 \pi r} \iint_{S_{a}}(\hat{\theta} \hat{\theta}+\hat{\phi} \hat{\phi}) \bullet \vec{M}(x, y) e^{j k_{o} \sin \theta(x \cos \phi+y \sin \phi)} d x d y
$$

\section{Effect of finite ground plane :}

The infinite ground plane solution described in the above sections yields the field radiated in the forward direction. This field is obtained by equation (11). The effect of finite ground plane is incorporated by employing the compact edge diffraction coefficients[7] and following the procedure described in [6]. The radiation fields are supplemented by the additional field contributions arising from the diffraction by the edges of the finite ground plane. The diffracted field supplements the field in the forward region whereas it accounts totally for the field in the shadow region(behind the finite ground plane) where the infinite ground plane solution gives a zero field.

The geometry of the finite ground plane is illustrated in figure 1. The mid 
points on the two edges of length $\mathrm{W}_{2}$ are given by $\mathrm{A}$ and $\mathrm{B}$, respectively, whereas the mid points on the two edges of length $W_{1}$ are given by $C$ and $D$, respectively. The diffracted fields at A and B are given by[6]

$$
\begin{aligned}
& H_{\theta A}^{d} \sim \frac{1}{2} H_{\theta f f}\left(d, \frac{\pi}{2}, 0\right) D_{s}\left(\rho_{c A}, \xi_{1}, \psi_{o}, \beta_{o}^{\prime}, n\right)\left[\frac{\rho_{c A}}{r\left(r+\rho_{c A}\right)}\right]^{1 / 2} \exp \left(-j k_{o} r\right) \exp \left(j k_{o} d \sin \theta\right)(12) \\
& H^{d}{ }_{\theta B} \sim \frac{1}{2} H_{\theta f f}\left(d, \frac{\pi}{2}, \pi\right) D_{s}\left(\rho_{c B}, \xi_{2}, \psi_{o}, \beta_{o}^{\prime}, n\right)\left[\frac{\rho_{c B}}{r\left(r+\rho_{c A}\right)}\right]^{1 / 2} \exp \left(-j k_{o} r\right) \exp \left(-j k_{o} d \sin \theta\right)(13)
\end{aligned}
$$

for $\phi$ polarization, and

$$
\begin{aligned}
& H_{\phi A}^{d} \sim \frac{1}{2} H_{\phi f f}\left(d, \frac{\pi}{2}, 0\right) D_{h}\left(\rho_{c A}, \xi_{1}, \psi_{o}, \beta_{o}^{\prime}, n\right)\left[\frac{\rho_{c A}}{r\left(r+\rho_{c A}\right)}\right]^{1 / 2} \exp \left(-j k_{o} r\right) \exp \left(j k_{o} d \sin \theta\right) \\
& H_{\phi B}^{d} \sim \frac{1}{2} H_{\phi f f}\left(d, \frac{\pi}{2}, \pi\right) D_{h}\left(\rho_{c B}, \xi_{2}, \psi_{o}, \beta_{o}^{\prime}, n\right)\left[\frac{\rho_{c B}}{r\left(r+\rho_{c A}\right)}\right]^{1 / 2} \exp \left(-j k_{o} r\right) \exp \left(-j k_{o} d \sin \theta\right)(1)
\end{aligned}
$$

for $\theta$ polarization. In the above equations,

$$
\rho_{c A}=\rho_{c B}=d=\frac{W_{1}}{2}
$$

and

$$
\begin{aligned}
& \xi_{1}=\frac{\pi}{2}+\theta \quad 0 \leq \theta \leq \pi \\
& \xi_{2}= \begin{cases}\frac{\pi}{2}-\theta & 0 \leq \theta \leq \frac{\pi}{2} \\
\frac{5 \pi}{2}-\theta & \frac{\pi}{2} \leq \theta \leq \pi\end{cases}
\end{aligned}
$$

Similarly, the diffracted fields at C and D are also calculated by replacing $\rho_{c C}=\rho_{c D}=d=\frac{W_{2}}{2}$. The detailed expressions for diffraction coefficients $D_{s / h}$ are 
given by [7, eq.113]. Total diffracted field is given by

$$
\vec{H}^{d}= \begin{cases}\vec{H}_{A}{ }^{d}+\vec{H}_{B}{ }^{d} & \phi=0^{\circ} \text { plane } \\ \vec{H}_{C}{ }^{d}+\vec{H}_{D}{ }^{d} & \phi=90^{\circ} \text { plane }\end{cases}
$$

The diffracted fields for $\phi=180^{\circ}$ and $\phi=270^{\circ}$ planes can also be calculated in a similar manner. The total radiated far field is given by adding the diffracted field and farfield magnetic field given in equation (25); i.e.,

$$
\vec{H}_{f f}^{t}=\vec{H}_{f f}+\vec{H}^{d}
$$

It should be noted that in the above analysis, the interaction between the aperture field and the diffracted field is ignored. It can be seen in the following section, that even with this approximation, the present analysis predicts the effect of finite ground plane on the radiation pattern with reasonable accuracy.

\section{NUMERICAL AND EXPERIMENTAL RESULTS}

To validate the present analysis various numerical examples are considered. As a simple case, open ended waveguide structures excited by a dominant mode field and radiating into half space through an infinite ground plane are analyzed. To study the effect of finite ground plane, a coaxial aperture in a finite ground plane and excited by a coaxially fed cavity is considered. The numerical data for these cases is compared with earlier published results and the experimental data obtained at NASALangley Research Center. 


\subsection{Apertures in Infinite Ground Plane::}

\subsubsection{Rectangular Aperture:}

A rectangular aperture as shown in figure $2 \mathrm{a}$ with dimensions $\mathrm{a}=4.755 \mathrm{~cm}$, $\mathrm{b}=2.215 \mathrm{~cm}$, and $\mathrm{L}=1.9 \mathrm{~cm}$ in an infinite ground plane and excited by a rectangular waveguide loaded with a dielectric $\operatorname{plug}\left(\varepsilon_{r}=3.76\right)$ is considered(figure 2(a)). The dielectric plug is discretized using tetrahedral elements. The air dielectric interface inside the waveguide is considered as input plane. The dominant $\mathrm{TE}_{10}$ mode is considered to be incident at the plane $\mathrm{z}=-\mathrm{L}$. The input admittance of this structure is calculated as a function of frequency and plotted in figure 2(b). The numerical results agrees very well with the data presented by Swift[12].

\subsubsection{Circular Aperture:}

A circular aperture in an infinite ground plane and excited by a circular waveguide is considered as a second example. The geometry is shown in figure 3(a). The circular waveguide of length $L=1 \lambda$ from the aperture is considered as a circular cavity for FEM discretization. The dominant $\mathrm{TE}_{11}$ mode is considered to be incident at the plane $\mathrm{z}=-\mathrm{L}$. The input admittance is computed as a function of the radius of the waveguide and plotted in figure 3(b) along with the numerical data given by Bailey and Swift[16]. Very good agreement is seen between the results computed using the present technique and those of [13].

\subsubsection{Coaxial Aperture:}

As a third example, a coaxial aperture excited by a coaxial line is considered. The geometry is shown in figure 4(a). Again the coaxial line of length $L=1 \lambda$ from the aperture is considered as a coaxial cavity for FEM discretization. Only dominant 
TEM mode is considered for both incident and reflected fields in the coaxial feed line as the higher order modes do not seem to be contributing much to the final result. The input admittance is computed as a function of frequency and plotted in figure 4(b). The results are compared with the available data in the literature[14].

\subsection{Apertures in Finite Ground Plane:}

To verify the validity of the current method when combined with GTD, a coaxial cavity with a finite ground plane is considered. The geometry of the cavity is shown in figure 5 . The cavity is fed by a coaxial line with the center conductor extending upto the radiating aperture. The cavity volume is discretized using 4474 tetrahedral elements, resulting in 4904 unknowns. For experimental verification, the cavity with dimensions as given in figure 5 is fabricated and its input return loss and radiation patterns as a function of frequency are measured at NASA-Langley Research Center. The input return loss computed using the present method assuming infinite ground plane is presented in figure 6 along with the experimental results. It can be noted that the finite ground plane in this case does not effect the input return loss properties.

The far field patterns using expression (30) for the structure shown in figure 5 are calculated for two frequencies, $f_{1}=5.0 \mathrm{GHz}$ and $f_{2}=9.0 \mathrm{GHz}$ and are shown in figures 7-8 along with the experimental data taken at NASA-Langley Research Center's low frequency Antenna Test Facility. Good agreement between the results obtained by the present analysis and the experimental data supports validity of present analysis. Since radiation pattern of an antenna depends upon the aperture field it is informative to know the tangential electric field over the aperture of the structure shown in figure 5 . In figures 9 the tangential electric field over the aperture is plotted for $f_{1}=5.0 \mathrm{GHz}$ and 
$f_{2}=9.0 \mathrm{GHz}$. As expected the aperture field deviates from the TEM type field as the frequency of operation increases.

\section{CONCLUSIONS}

Radiation characteristics of cavity backed aperture antennas in finite ground plane are presented using a hybrid FEM/MoM technique and GTD. Various numerical examples are considered to demonstrate the flexibility and validity of the method. Very good agreement is seen between the numerical data obtained using the present method and the data available in the literature. The successful application of the GTD method in conjunction with FEM-MoM is demonstrated for calculating the radiation patterns of a coaxial cavity with finite ground plane. Since FEM is used for cavity region this method is applicable to arbitrarily shaped cavities with complex and anisotropic materials. By proper modelling, the present method can be used to analyze microstrip antenna arrays residing in a cavity with finite ground plane. This method can also be easily extended to a finite circular ground plane without much effort.

\section{ACKNOWLEDGEMENTS}

The authors would like to thank Mr. Carl A. Lipp, Mr. Robert Holt and Mr. Hunter Walden for fabricating the coaxial cavity and performing the experiments. 


\section{REFERENCES}

[1] J.M.Jin and J.L.Volakis, "A hybrid finite element method for scattering and radiation by microstrip antennas and arrays residing in a cavity," IEEE Trans. Antennas and Propagation, Vol.AP-39, pp.1598-1604, Nov. 1991.

[2] A. Chatterjee, J.Jin and J.L.Volakis, "Edge based finite elements and vector ABC's applied to 3D scattering," IEEE Trans. Antennas and Propagation, Vol.AP-41, pp.221226 , Feb. 1993.

[3] J.M.Jin and J.L. Volakis, "A finite element-boundary integral formulation for scattering by three dimensional cavity backed apertures," IEEE Trans. Antennas and Propagation, Vol.AP-39, pp.97-104, Jan. 1991.

[4] X.Yuan, "Three dimensional electromagnetic scattering from inhomogeneous objects by hybrid moment and finite element method," IEEE Trans. Microwave Theory and Techniques, Vol. MTT-38, pp.1053-1058, Aug. 1990.

[5] J.Jin, The Finite Element Method in Electromagnetics, John Wiley \& Sons, Inc., New York, 1993.

[6] C.R.Cockrell and P.H.Pathak, "Diffraction theory techniques applied to aperture antennas on finite circular and square ground planes," IEEE Trans. Antennas and Propagation, Vol.AP-22, pp.443-448, May 1974.

[7] P.H.Pathak and R.G. Kouyoumjian, "The dyadic diffraction coefficient for a perfectly conducting wedge," ElectroScience Lab., Ohio State Univ., Columbus, Rep. 21834, June 5,1970, Contract No. AF19(628)-5929.

[8] S.M.Rao, D.R.Wilton and A.W.Glisson, "Electromagnetic scattering by surfaces of arbitrary shape," IEEE Trans. Antennas and Propagation, Vol.AP-30, pp.409-418, May 1982.

[9] R.F.Harrington, Time Harmonic Electromagnetic Fields, McGraw Hill Inc, 1961.

[10] C.J.Reddy, M.D.Deshpande, C.R.Cockrell and F.B.Beck, "Analysis of three-dimensional-cavity-backed-aperture antennas using a combined finite element method/ method of moments/geometrical theory of diffraction technique," NASA Technical Paper 3548, November 1995. 
[11] T.Sarkar, K.Wang, E.Arvas, "A limited survey of various conjugate gradient methods for solving complex matrix equations arising in electromagnetic interations," Wave Motion, Vol.10, pp.527-546, 1988.

[12] C.T.Swift, "Admittance of a waveguide fed aperture loaded with a dielectric plug," IEEE Trans. on Antennas and Propagation, Vol.AP-18, pp.356-359, May 1969.

[13] M.C.Bailey and C.T.Swift, "Input admittance of a circular waveguide aperture covered by a dielectric slab," IEEE Trans. on Antennas and Propagation, Vol.AP-17, pp.386-391, July 1968.

[14] C.T.Swift, "Input admittance of a coaxial transmission line opening onto a flat, dielectric covered ground plane," NASA Technical Report NASA TND-4158, 1967. 


\section{LIST OF FIGURES}

Figure 1 Geometry of a cavity backed aperture in finite ground plane.

Figure 2 (a) Rectangular waveguide with a dielectric plug radiating into half space. $a=4.755 \mathrm{~cm}$., $b=2.215 \mathrm{~cm}$., $L=1.9 \mathrm{~cm}$., $\varepsilon_{r}=3.76$

(b) Variation of normalized admittance of the above structure with frequency. The solid and dashed lines are computed using the present analysis and the solid circles are from Swift[12].

Figure 3 (a) Circular waveguide with radius $r$ radiating into half space. $\mathrm{L}=1.0 \lambda, \varepsilon_{r}=1.0$

(b) Input admittance of the above structure as a function of $2 r / \lambda$. The solid and dashed lines are computed using the present analysis and the solid circles are from Bailey and Swift[13]

Figure 4 (a) Coaxial line with inner radius $r_{1}$ and outer radius $r_{2}$ radiating into half space. $r_{2} / r_{1}=1.57, \mathrm{~L}=1.0 \lambda, \varepsilon_{r}=1.0$

(b) Input admittance as a function of $k_{o} r_{1}$. The solid lines are computed from the present analysis and the solid circles are from Swift[14]

Figure 5 Geometry of a coaxial cavity with finite rectangular ground plane. $\mathrm{W}_{1}=24^{\prime \prime}$, $\mathrm{W}_{2}=12^{\prime \prime}, \mathrm{L}=3 / 8^{\prime \prime}$. Outer radius of the coaxial cavity $=1^{\prime \prime}$, Inner radius of the coaxial cavity $=0.0181^{\prime \prime}$. The cavity is fed by a $50 \Omega$ coaxial line.

Figure 6 Return loss of the coaxial cavity with frequency. The solid line is computed using the present analysis and the solid circles are the experimental results.

Figure 7 (a) Radiation pattern of the coaxial cavity at $5 \mathrm{GHz}$ at $\phi=0^{\circ}$ and $\phi=180^{\circ}$

(b) Radiation pattern of the coaxial cavity at $5 \mathrm{GHz}$ at $\phi=90^{\circ}$ and $\phi=270^{\circ}$

This method with GTD, - - - - - - This method with infinite ground plane, - . - . - . Experiment. 
Figure 8 (a) Radiation pattern of the coaxial cavity at $9 \mathrm{GHz}$ at $\phi=0^{\circ}$ and $\phi=180^{\circ}$.

(b) Radiation pattern of the coaxial cavity at $9 \mathrm{GHz} i \phi=90^{\circ}$ and $\phi=270^{\circ}$.

This method with GTD, - - - - - - This method with infinite ground plane, $\ldots \cdot \cdot \cdot-\cdot \cdot-$ Experiment.

Figure 9 The vector plot of the electric field at the aperture of the coaxial cavity. The amplitudes have been equalized for clarity of presentation.

(a) $5 \mathrm{GHz}$ (b) $9 \mathrm{GHz}$. 

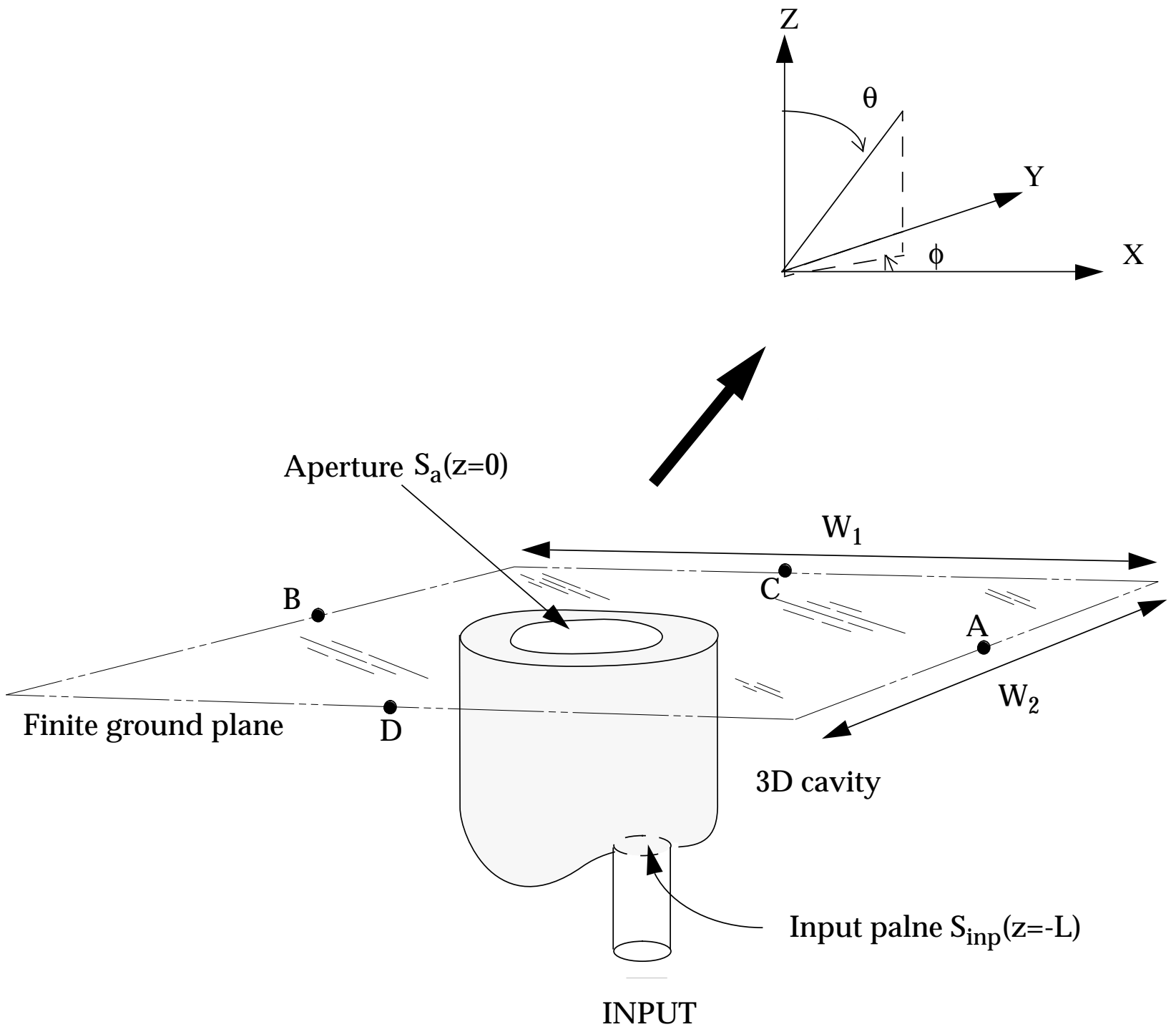

Figure 1 


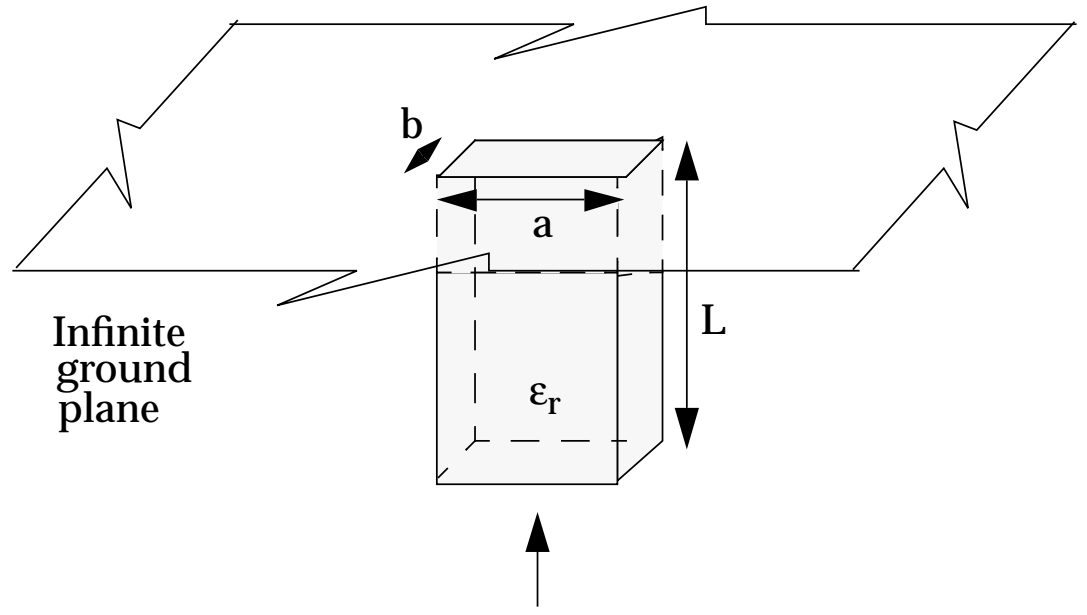

Input

(a)

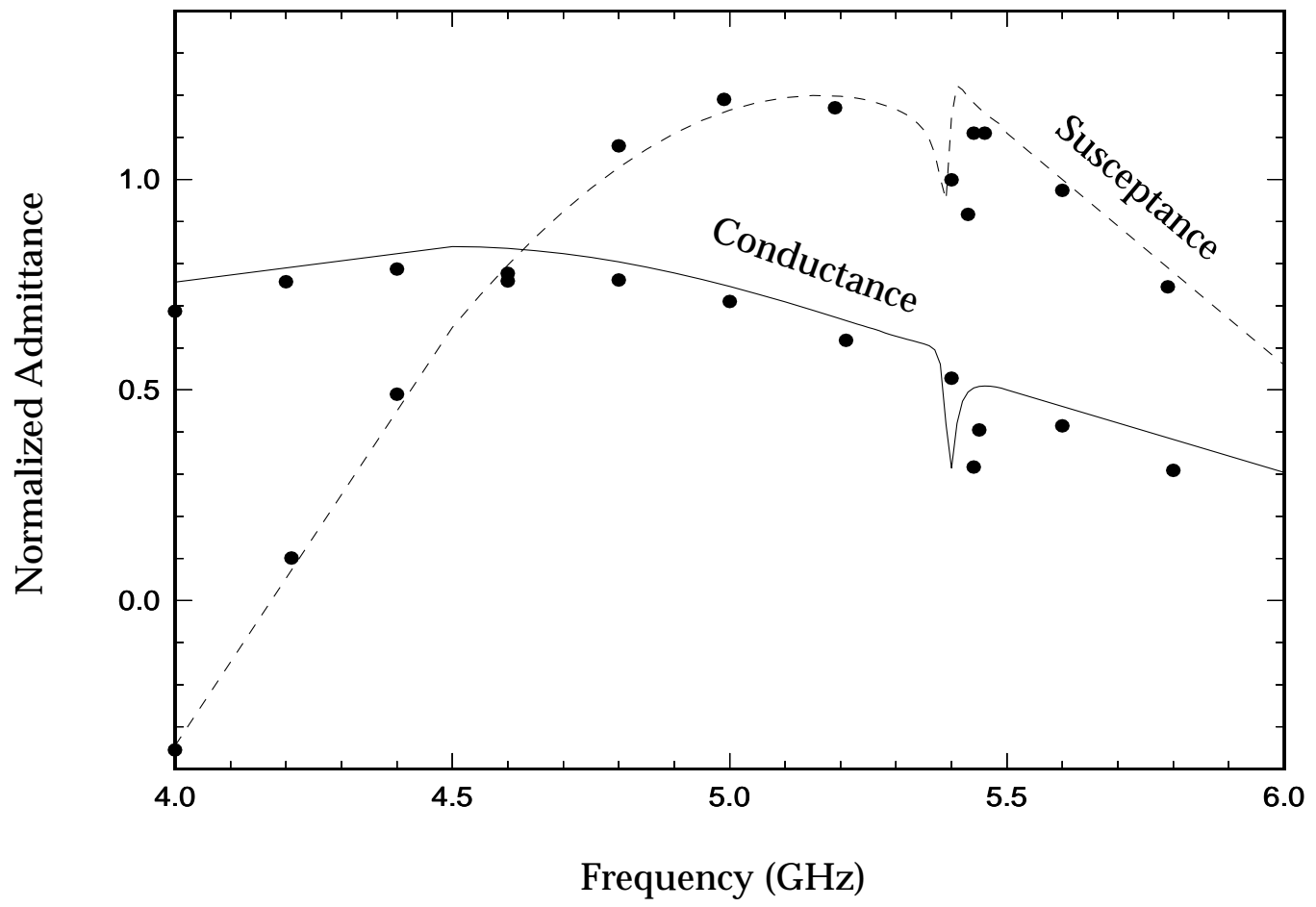

(b)

Figure 2 


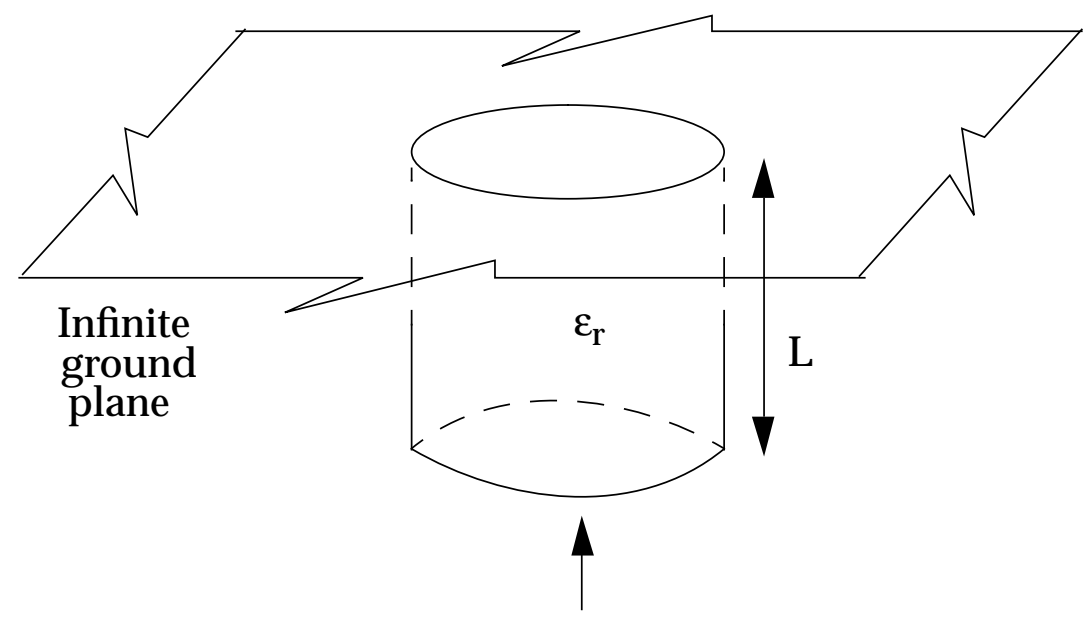

Input

(a)

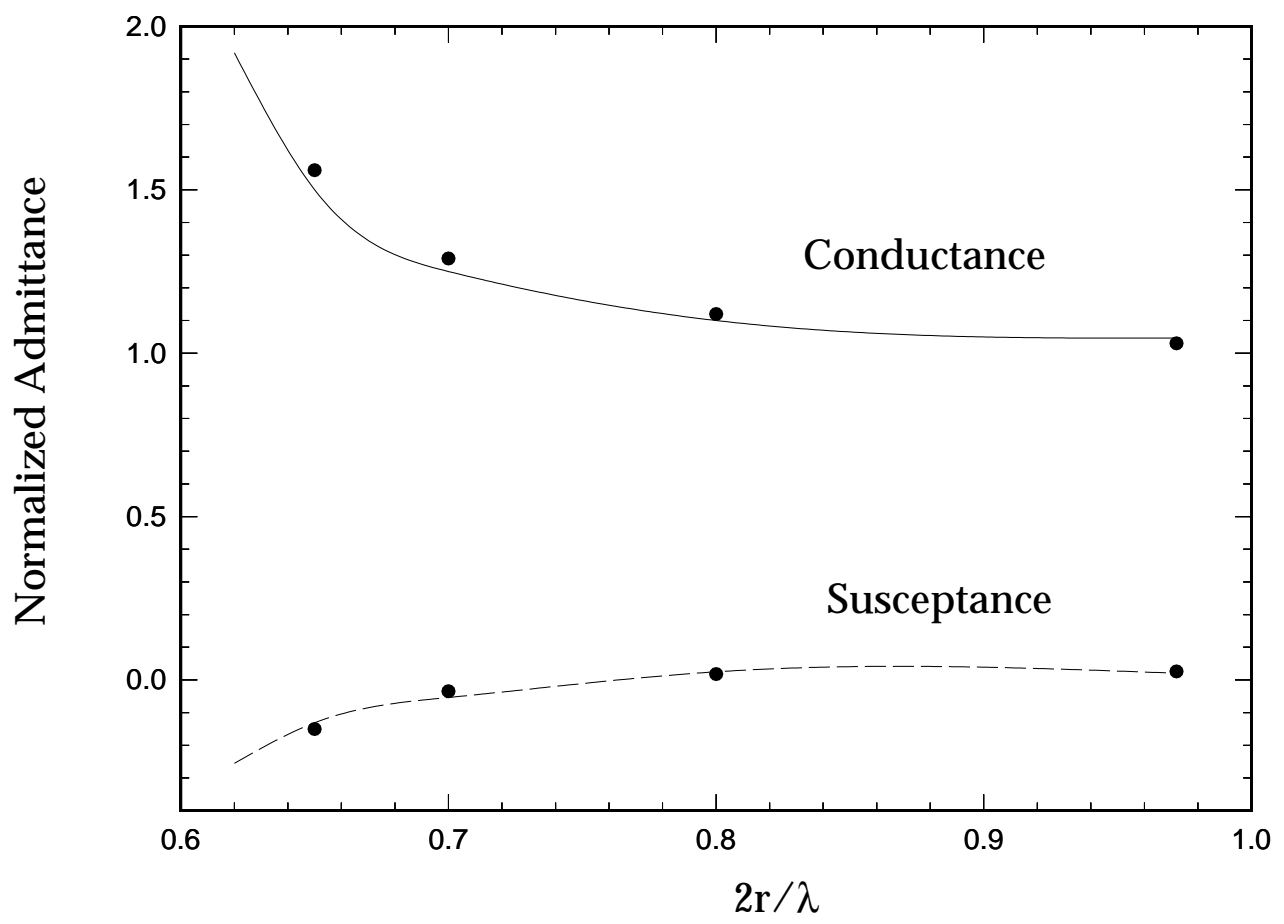

(b)

Figure 3 


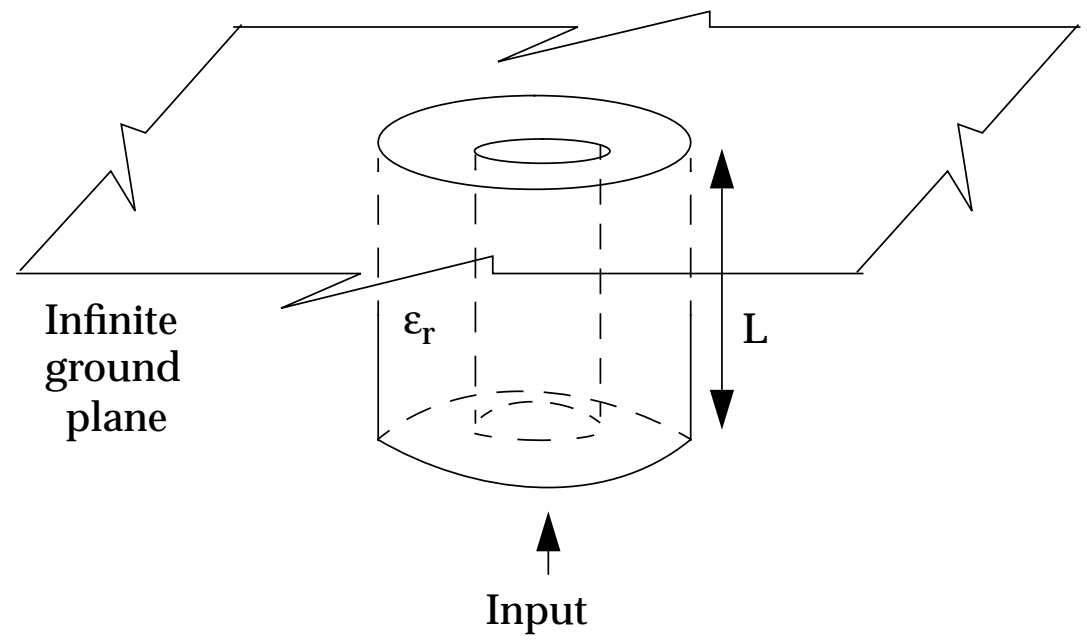

(a)

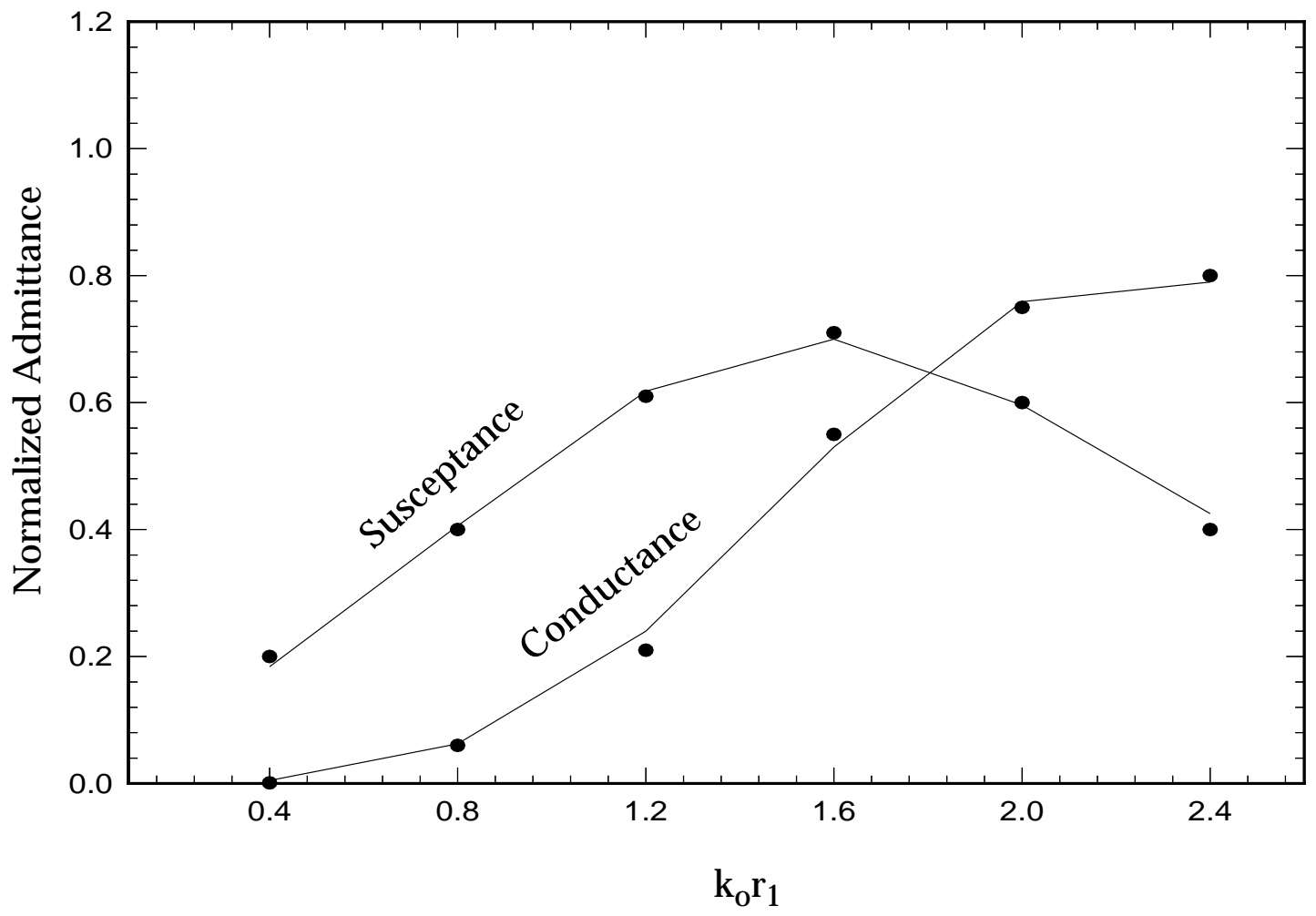

(b)

Figure 4 


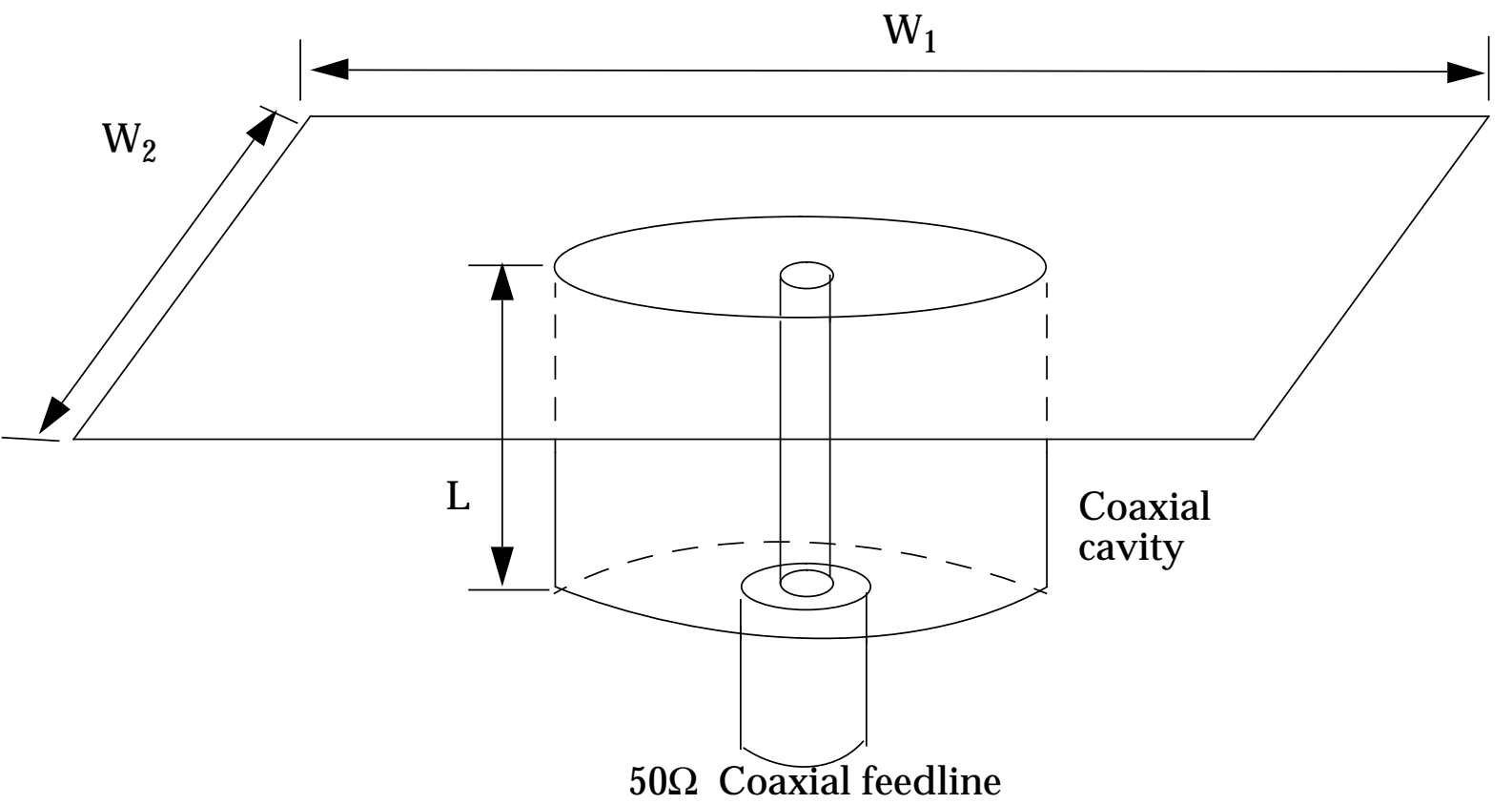

Figure 5 


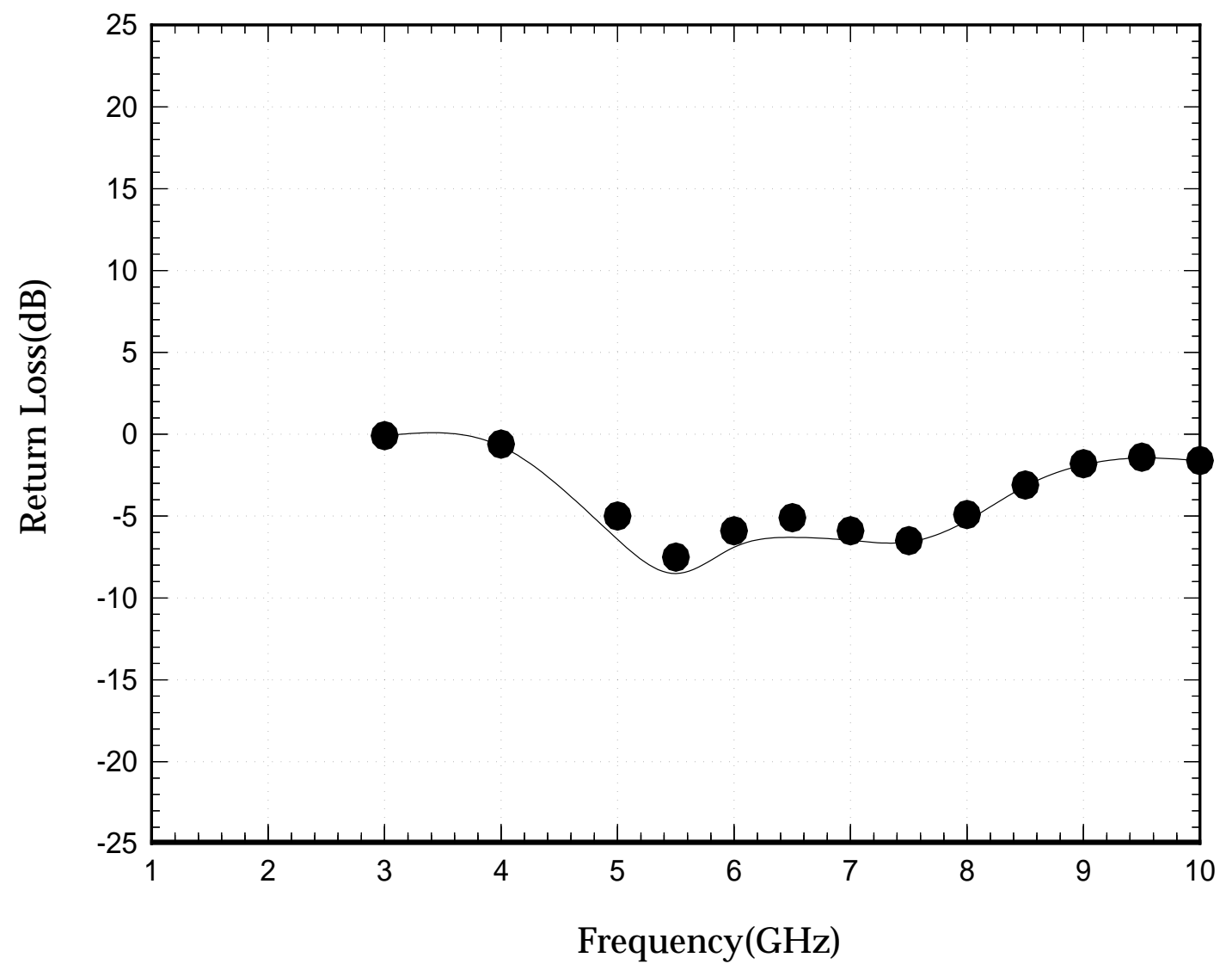

Figure 6 


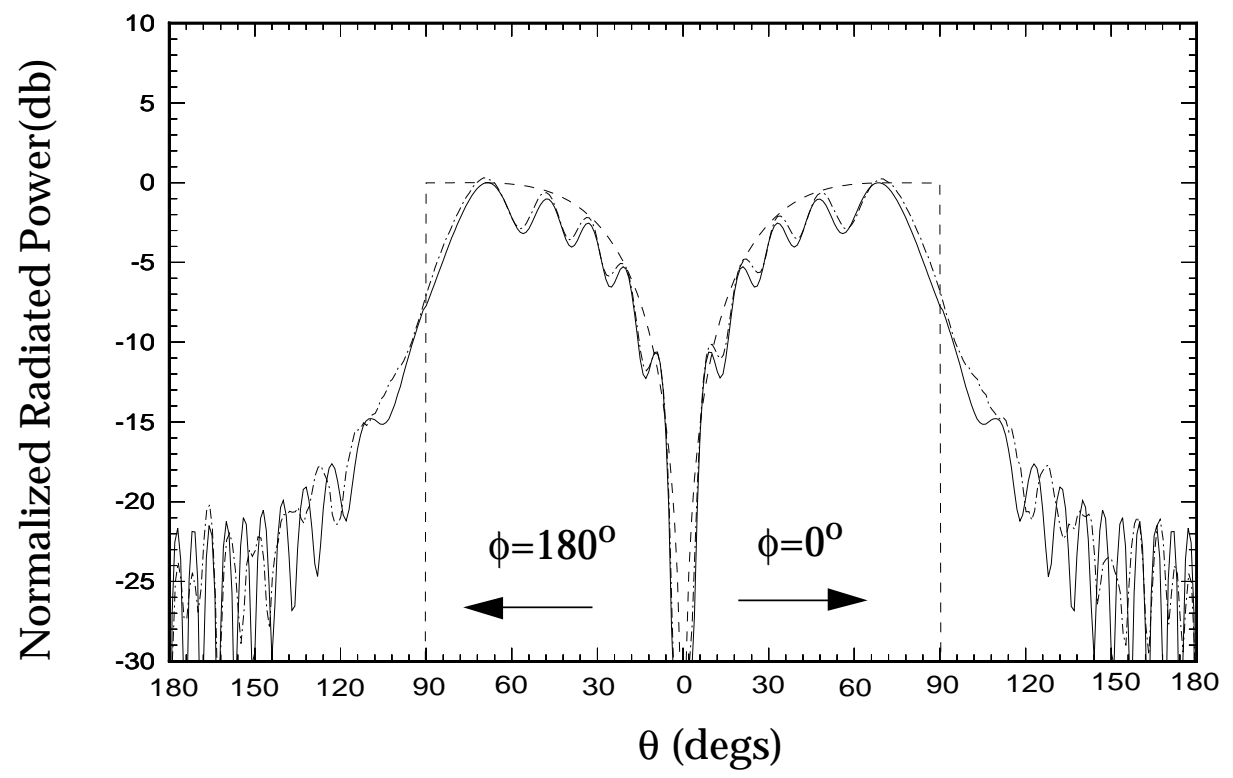

(a)

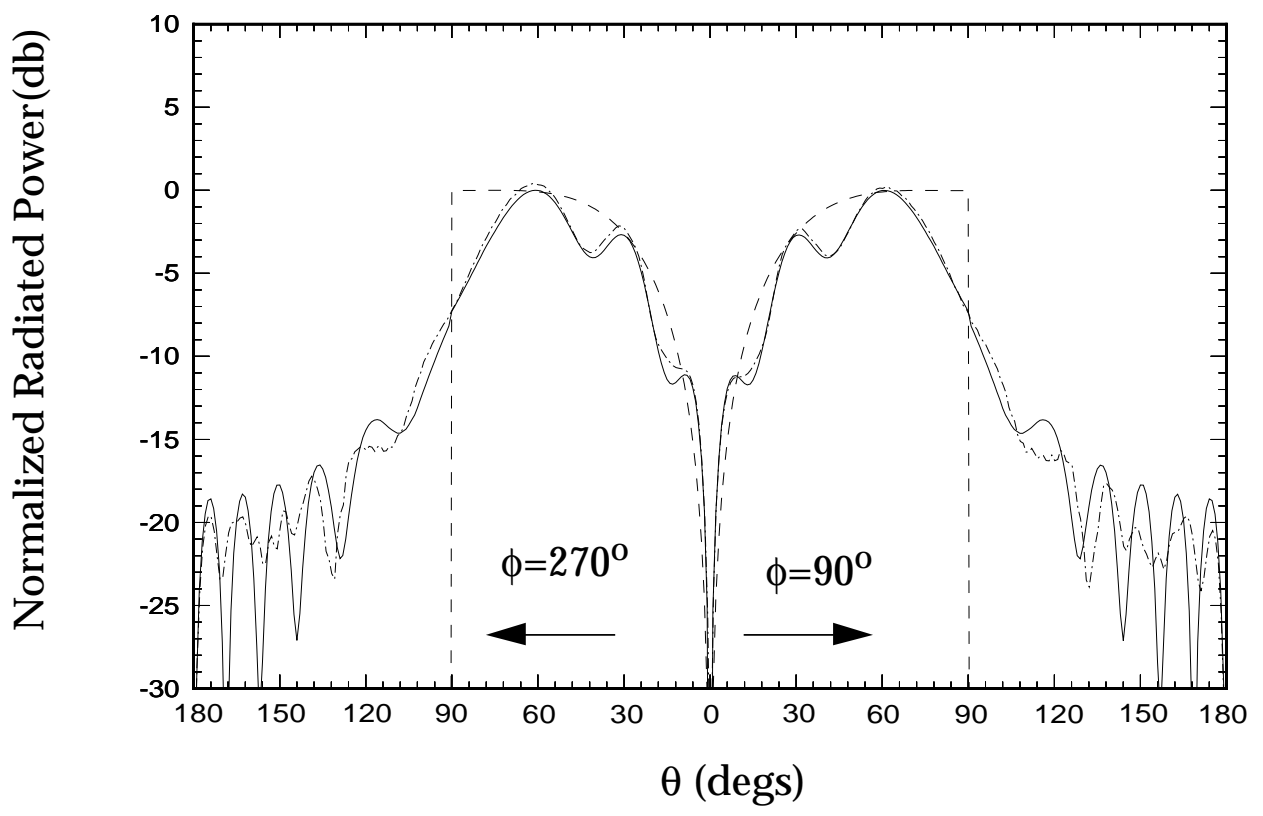

(b)

Figure 7 


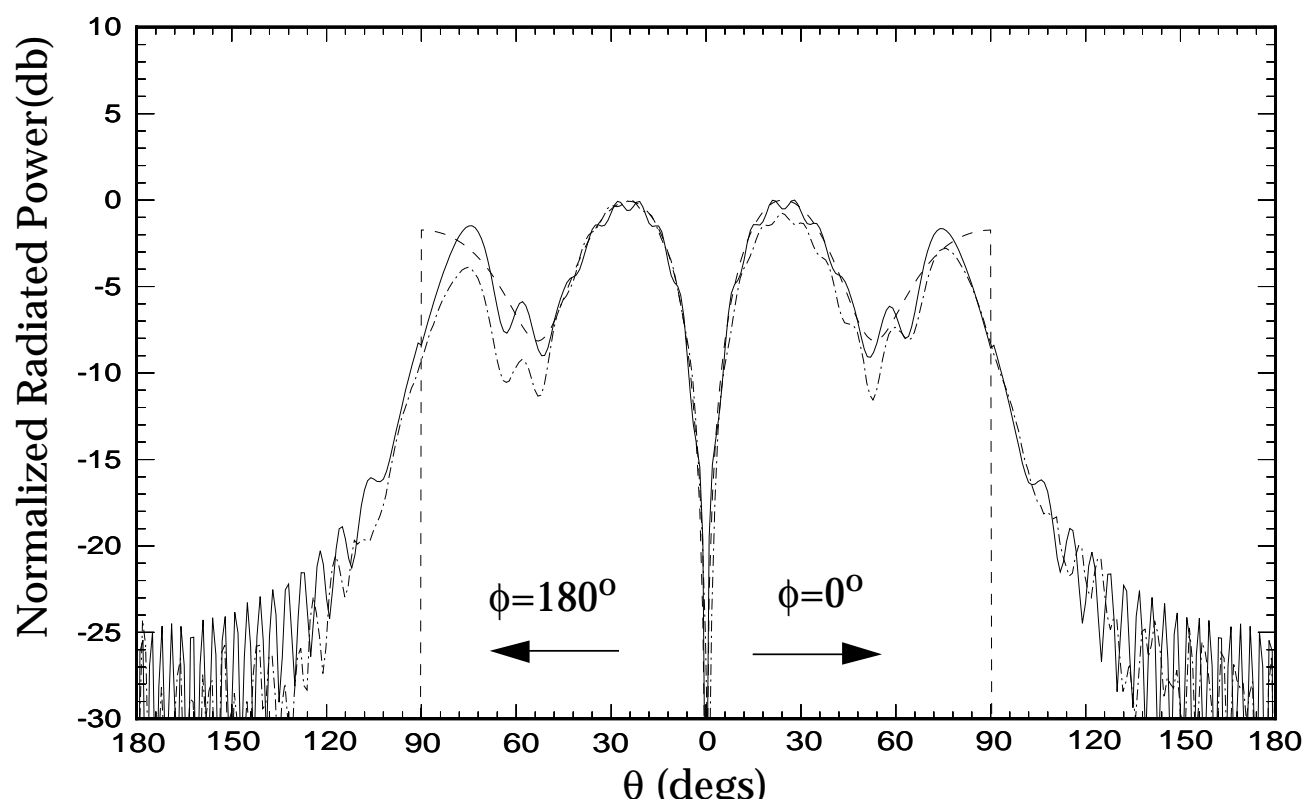

(a)

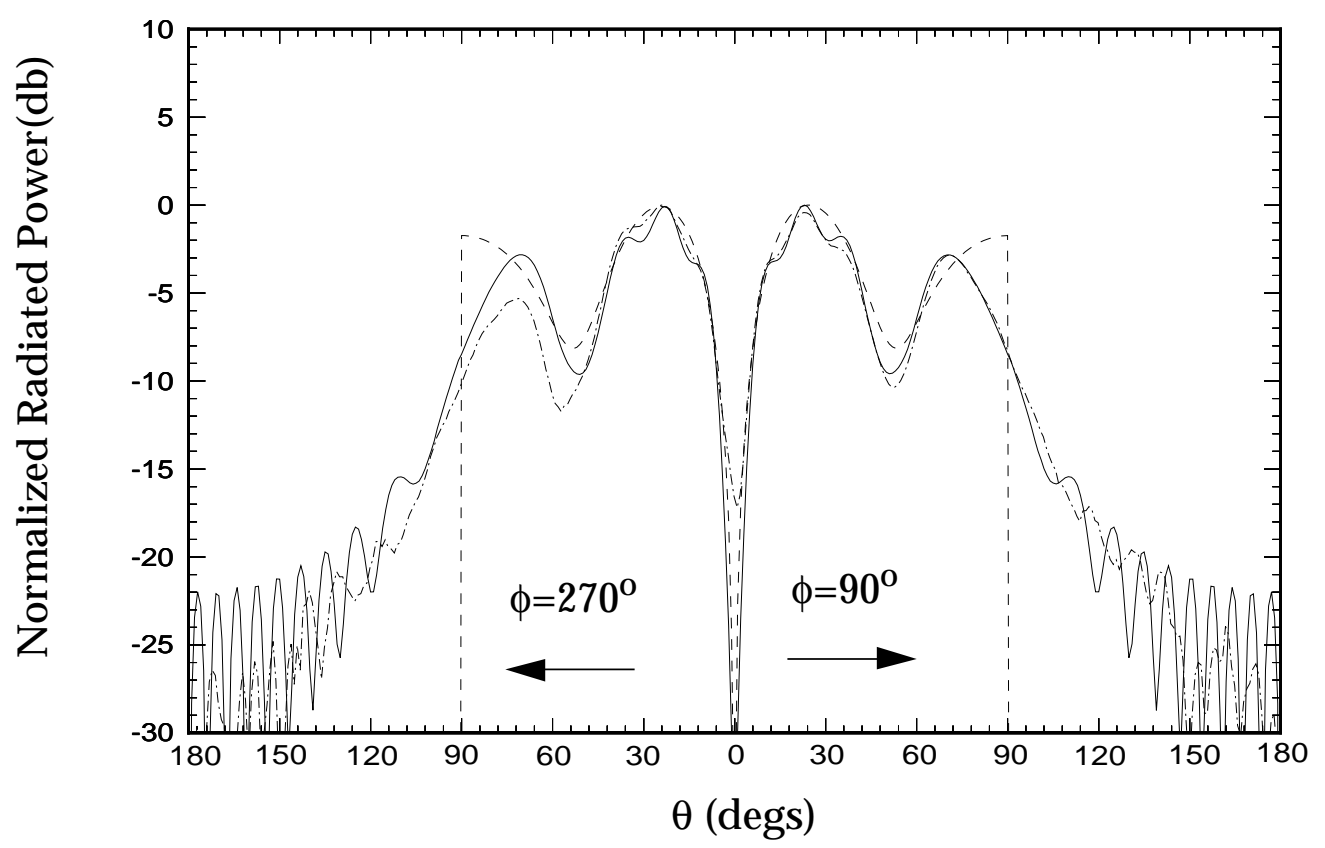

(b)

Figure 8 


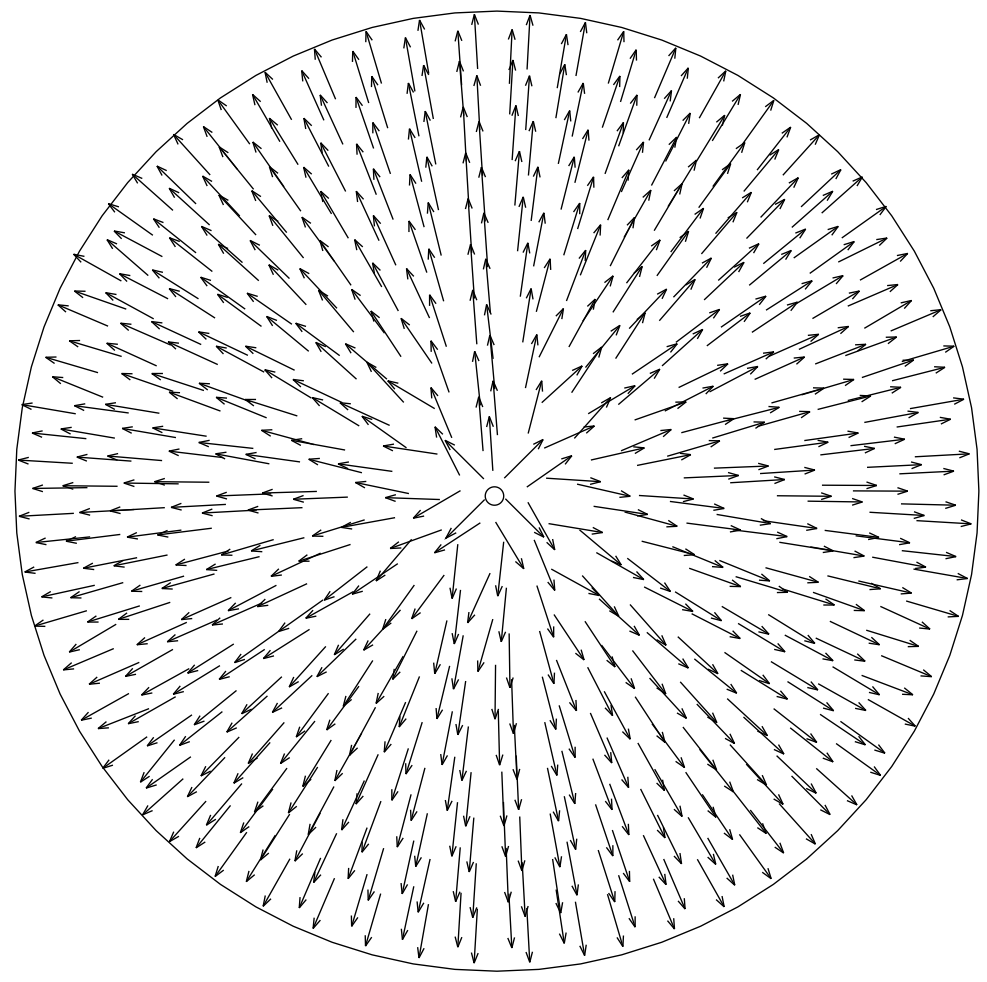

Figure 9(a) 


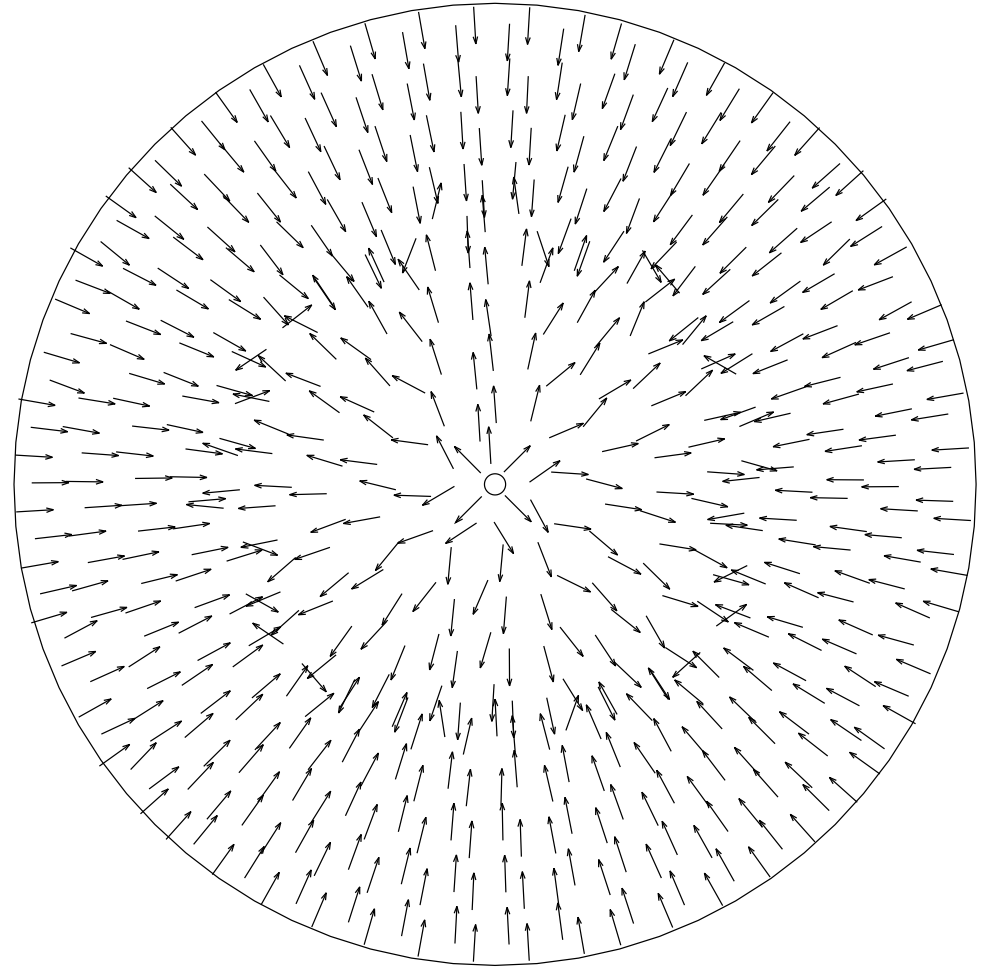

Figure 9(b) 